\title{
Belphégor
}

\section{Roger Bozzetto, Mondes fantastiques et réalités de l'imaginaire}

\section{Vittorio Frigerio}

\section{Q OpenEdition}

1 Journals

\section{Electronic version}

URL: http://journals.openedition.org/belphegor/716

DOI: 10.4000/belphegor.716

ISSN: 1499-7185

\section{Publisher}

LPCM

\section{Electronic reference}

Vittorio Frigerio, «Roger Bozzetto, Mondes fantastiques et réalités de l'imaginaire », Belphégor [Online], 14 | 2016, Online since 16 July 2016, connection on 24 September 2020. URL : http:// journals.openedition.org/belphegor/716 ; DOI : https://doi.org/10.4000/belphegor.716

This text was automatically generated on 24 September 2020.

\section{(c) (i) (9)}

Belphégor est mis à disposition selon les termes de la Licence Creative Commons Attribution - Pas d'Utilisation Commerciale - Pas de Modification 4.0 International. 


\title{
Roger Bozzetto, Mondes fantastiques et réalités de l'imaginaire
}

\author{
Vittorio Frigerio
}

\section{REFERENCES}

Bozzetto, Roger. Mondes fantastiques et réalités de l'imaginaire, Aix-en-Provence : Presses Universitaires de Provence. Collection « Regards sur les fantastiques », 2015. 226 pp.. ISBN : 9782853999557. 


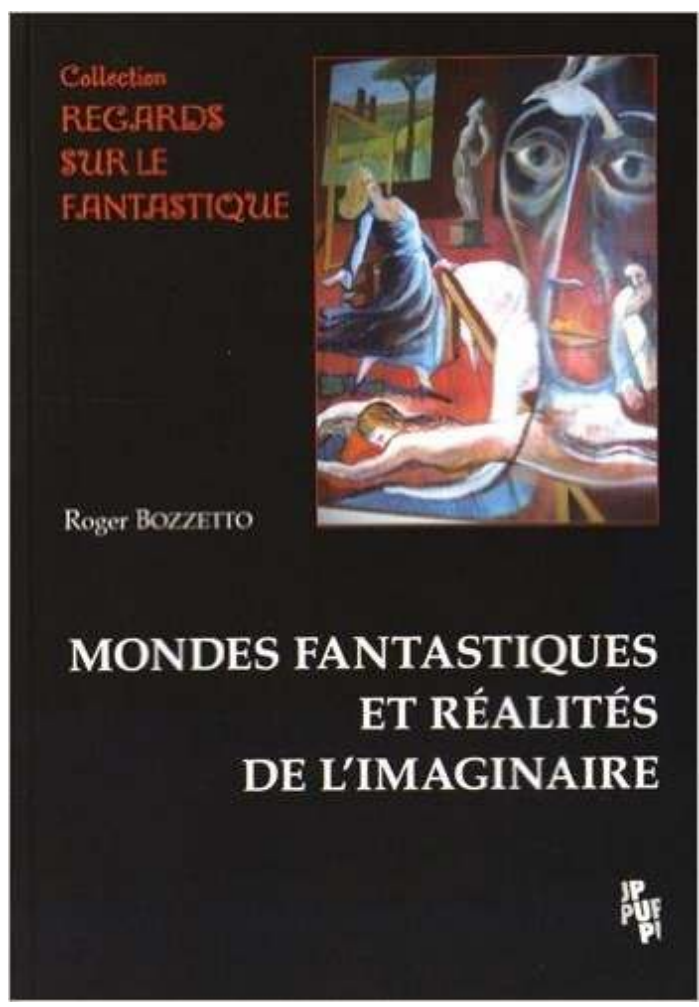

1 Roger Bozzetto est un des critiques les plus actifs, et les plus habituellement engageants, dans le domaine de la littérature fantastique, à laquelle il a déjà consacré nombre de travaux importants. On ne devrait donc pas s'étonner de voir paraître maintenant un nouvel ouvrage, qui reprend les préoccupations qui ont toujours été les siennes en les élargissant ultérieurement. La réflexion sur la nature, l'étendue et les modalités du fantastique se marient ici à des explorations dans le champ de la sciencefiction, de la fantasy, ce genre éminemment anglo-saxon qui n'a même pas de traduction française, et à des percées dans les territoires de chasse de la littérature " générale " - celle à laquelle on n'a pas collé d'étiquette, ou qui n'est pas, pour reprendre l'expression de Bozzetto lui-même, «marquée ». L'exploration des territoires inquiétants du fantastique s'ancre dans une mise en contexte historique qui offre l'occasion à l'auteur de réfléchir à loisir sur les particularités sociales et idéologiques de notre époque, comparée à celle qui a vu le premier grand essor de ce type de littérature. Bozzetto croit identifier dans les temps que nous vivons les traces de transformations essentielles, qui font que «nous sommes en train de changer d'univers mental » (p. 12). Transformations, celles-ci, que sous d'autres formes et dans d'autres conditions avaient pu sentir aussi les auteurs dont les travaux ont fourni les lettres de gloire de ce genre si difficile à cerner. Une bonne partie de l'analyse du critique part de la redécouverte encore récente de la présence de la notion de "genre fantastique " en littérature déjà dans l'Encyclopédie de Diderot et D'Alembert, sous la signature de Marmontel - ce qui vieillit d'une centaine d'années en tout cas la notion et justifie « un retour en amont [...] qui oblige à repenser des notions que l'on croyait maîtriser» (p. 22). Ce retour en amont est également et heureusement complété par une descente en aval, qui mène l'auteur à redécouvrir d'autres détails intéressants de la critique traditionnelle du fantastique, en revisitant par exemple l'œuvre très connue de Caillois pour en exhumer la notion relativement secondaire d' " apparition ", présentée comme une clef de lecture susceptible de révéler des modes de fonctionnement sous- 
estimés du genre. Et aussi à pousser beaucoup plus loin, discutant de nombre d'auteurs de science-fiction contemporaine (en particulier Kim Stanley Robinson). Bozzetto réussit ainsi à ébaucher des voies nouvelles possibles, en mettant côte à côte des notions proposées par d'autres critiques, et suggérant qu' « [u]ne relecture des textes canoniques qui prendrait en compte les notions d'apparition, de sidération, de monstration pourrait permettre de questionner les présupposés de la tradition standard, et faire surgir de nouvelles pistes, enrichissant notre compréhension du fantastique et de ses effets » (p. 31).

Un des aspects les plus intéressants de l'ouvrage vient justement de la volonté de l'auteur de dépasser les frontières traditionnelles entre la littérature sans étiquette et celle de genre, qui lui permet de discuter les uns à la suite des autres d'auteurs tels que Faulkner, Stevenson, Tolkien, Borges, Lovecraft, Jean Ray ou Stephen King. Une lecture très attentive est consacrée à ce petit chef d'œuvre trop peu connu qu'est L'Invention de Morel d'Adolfo Bioy-Casares. Bozzetto ne peut également s'empêcher de revenir sur certains des romanciers qu'il a le plus souvent traités, notamment Jules Verne ou J.G. Ballard. On profite avec plaisir des petites illuminations que partage ainsi un lecteur vorace, doué d'une connaissance encyclopédique de son sujet. Parmi les diverses surprises agréables, notons une discussion simple et juste sur les rapports entre le western et la science-fiction.

3 L'intérêt du livre, indéniable pour les passionnés du sujet, est toutefois sensiblement mitigé par la qualité (ou plutôt l'absence de qualité) de la révision du texte. Les coquilles abondent. Il y a ici et là, beaucoup plus souvent qu'on ne s'y attendrait pour des presses universitaires, des mots manquants, des phrases bancales, des liens logiques qui restent à deviner. Cela va jusqu'à retrouver, l'un à la suite de l'autre, comme à la page 187 , deux paragraphes qui sont visiblement deux versions légèrement différentes du même texte, dont l'ancienne s'est débrouillée pour survivre on ne sait trop comment à un nettoyage clairement très sommaire. La patience du lecteur s'en trouve par moments mise à dure épreuve. Une note à la toute fin de l'ouvrage indique bien que « ce volume est composé en partie par des textes publiés ailleurs et remaniés " (sans que l'on indique nulle part, d'ailleurs, ni où ni quand les premières publications auraient eu lieu). Il n'en demeure pas moins qu'on se retrouve quelque peu dérouté, par exemple lorsqu'en lisant la section consacrée à Ursula Le Guin on se rend compte qu'elle est basée sur une comparaison avec une autre auteure dont le nom est tu. Il y est question des différences entre les «deux écrivaines» (p. 112), mais une seule d'entre elles a droit de cité, et encore, sans références critiques en dépit de l'abondance de travaux qui ont été consacrés à cette voix importante de la science-fiction américaine. On ne peut conclure de ceci qu'un seul fait déplorable : le réviseur était manifestement aux abonnés absents. C'est inadmissible pour une maison d'édition universitaire, mais c'est surtout malheureux, car cela ôte de la crédibilité à un volume qui est pourtant riche de remarques très pertinentes et qui méritait un bien meilleur traitement. 


\section{AUTHOR}

VITTORIO FRIGERIO

Dalhousie University 